\title{
GOZ-Tipp \\ Begründung wird einfach abgelehnt: Erläuterung hilft
}

\author{
Ein Patient ist verärgert wegen einer abgelehnten Begründung für eine bestimmte Behandlung. \\ Ärgerlich auf wen? Auf die Beihilfe, die nicht zahlt? Oder auf den Zahnarzt? Wenn er sich über \\ die Beihilfe ärgert, dann ist das berechtigt. Denn eine verordnungskonforme Begründung zu be- \\ anstanden, also zahnmedizinisch-inhaltlich anzuzweifeln oder gar als nicht zutreffend zu be- \\ zeichnen, steht einem Beihilfesachbearbeiter nicht zu, ist meist anmaßend und gegebenenfalls \\ sogar ein nicht hinnehmbarer rechtsrelevanter Übergriff.
}

Zu zahnmedizinisch-inhaltlicher Prüfung ist der Laie „Sachbearbeiter" nicht befähigt, und es bedarf in der Regel zahnärztlichen Sachverstands, zum Beispiel eines Gutachters oder der Zahnärztekammer. Formale Fehler, wie eine fehlende Begründung, das Bemessen unzulässiger Kriterien statt Schwierigkeit, Zeitaufwand und Umstände oder eine „Begründung“ ohne jeden konkreten Grund, kann der Sachbearbeiter allerdings aufzeigen.

Wenn der Patient auf den Zahnarzt ärgerlich ist? Dann hat der wohl im Vorfeld zumindest einen Fehler gemacht. Denn ein bestimmter Hinweis ist gemäß der üblichen Vorgehensweise der Beihilfe und auch der Postbeamtenkrankenkasse mittlerweile obligat: der Hinweis, dass bestimmte Beihilfestellen, Post et cetera prinzipiell versuchen, jedwede Erstattung über den Durchschnittsatz hinaus vorzuenthalten, egal mit welchen Argumenten. Gemäß Patientenrechtegesetz könnte dann sogar eine Verpflichtung gesehen werden, den Berechtigten auf das übliche Standardvorgehen der Verweigerung jedweder Erstattung oberhalb des Durchschnittsatzes vorab aufzuklären.

\section{Aufklärungsverpflichtung über routinemäßige "Nichtanerkennung"}

Wenn das versäumt wurde und der Patient ärgerlich ist, muss der Zahnarzt diese Aufklärung wenigstens nachholen und zusätzlich darauf verweisen, dass bis auf wenige ab 2012 höher bewertete Gebührenziffern ansonsten seit 1987 keine Gebührenerhöhung erfolgt ist. Mit fast drei Jahrzehnten stagnierender Vergütung ist eine den aktuellen Möglichkeiten der Zahnmedizin voll entsprechende Behandlung mit dem dazu nötigen Zeitaufwand unter Einsatz moderner Behandlungsmaterialien und -techniken stellenweise kaum mehr möglich. Wenn eine derartige Behandlung aber erfolgt ist - oder erfolgen soll -, muss dies mittels Faktorerhöhung berechnet werden.

\section{Was ist nach dieser Aufklärung zu tun?}

Am besten hält sich der Zahnarzt ohne Zögern und Aufschub strikt an die Rechtslage: Diese sieht vor, dass der Rechnung ausstellende Zahnarzt auf Verlangen des Zahlungspflichtigen die schriftlich abgegebene Begründung erläutert. So steht es unmissverständlich in $\$ 10$ Abs. 3 Satz 2 GOZ. Das ist eine Verpflichtung des Rechnungsausstellers, und ohne dieser nachzukommen, wird die über den Durchschnittssatz hinausgehende Vergütung nicht fällig. Da erhebt sich allerdings sofort die Fra- ge, warum bezüglich der Begründungen erstatterseitig derart herumargumentiert wird, oft seitenlang mit nicht, kaum oder nur stellenweise zutreffendem Textbausteinschutt und -schrott, anstatt dem Beihilfeberechtigten klipp und klar zu sagen: „Wir haben die Begründung nicht verstanden. Lassen Sie sich bitte die Begründung erläutern, wie es $\$ 10$ (3) 2 GOZ vorsieht. Dann können wir beihilfeseitig höher erstatten."

Derartige Hinweise an den Berechtigten erfolgen fast nie. Es ist nicht zu hoch gegriffen, das bewusste Verschweigen der Rechtslage als eine „Amtspflichtverletzung“ gemäß BGH-Urteil zu bezeichnen. Warum aber wird der Hinweis auf die Erläuterungspflicht seitens der Erstatter systematisch unterlassen? Weil ja dann der Berechtigte auf die Idee käme, tatsächlich Erläuterungen einzuholen, um dann gegenüber der Beihilfe zu erklären, nun sei die in der GOZ vorgeschriebene Formalie erfolgt, also ab jetzt Zahlung und Erstattung fällig.

\section{Erläuterungsbeispiel drastisch-plastisch, aber Alltag}

Was ist zu tun? Ohne Zögern die Begründung erläutern, am besten schriftlich mit dem Hinweis, dass jedenfalls der Rechnungsaussteller dem Berechtigten zur Seite steht. Erläutern ist einfach: ein laienverständliches, nachvollziehbares Erklären der auf der Rechnung genannten Gründe. Es werden die tatsächlichen Gründe mit einfachen Worten erneut etwas ausführlicher dargelegt, was sie konkret bedeuten für die Behandlung, die Behandlungsdurchführung und für den Behandler. Die Begründung „weit überdurchschnittliche Schwierigkeit wegen permanent hohem Zungendruck " bedeutet, dass die übertrainierte Zunge bereits anatomisch vergrößert ist, daher die Sicht auf die Unterkieferbezahnung ständig verdeckt und dem Oberkiefer außer bei maximaler, kaum länger durchzuhaltender Öffnungsweite ebenfalls anliegt und beim Abhalten unwillkürlich Gegendruck aufgebaut wird, der dann bei verstärktem Bemühen um Zungenverdrängung entweder zu reflektorischer Verringerung der Mundöffnung oder zu Würgereiz führt mit der Folge, dass die Weiterbehandlung häufig und zeitaufwändig unterbrochen werden muss.

So gut erklärt, kommt der Erstatter im Grunde nicht daran vorbei, auch tatsächlich eine Erstattung vorzunehmen. Zumindest aber hat die Praxis damit alle ihre rechtlichen Verpflichtungen erfüllt, insbesondere wenn der Zahlungspflichtige erklärt, dass er die Erläuterung verstanden hat. $\quad$ O ZAeG, P.E. 